\title{
Randomized, placebo-controlled window trial of EGFR, Src, or combined blockade in head and neck cancer
}

Julie E. Bauman, ${ }^{1}$ Umamaheswar Duvvuri, ${ }^{2}$ William E. Gooding, ${ }^{3}$ Tanya J. Rath, ${ }^{4}$ Neil D. Gross, ${ }^{5}$ John Song, ${ }^{6}$ Antonio Jimeno, ${ }^{7}$ Wendell C. Yarbrough, ${ }^{8}$ Faye M. Johnson, ${ }^{9}$ Lin Wang, ${ }^{10}$ Simion Chiosea, ${ }^{10}$ Malabika Sen, ${ }^{2}$ Jason Kass, ${ }^{2}$ Jonas T. Johnson, ${ }^{2}$ Robert L. Ferris, ${ }^{2}$ Seungwon Kim, ${ }^{2}$ Fred R. Hirsch, ${ }^{11}$ Kimberly Ellison, ${ }^{11}$ John T. Flaherty, ${ }^{1}$ Gordon B. Mills, ${ }^{12}$ and Jennifer R. Grandis ${ }^{2}$ 'Department of Medicine and 2Department of Otolaryngology, University of Pittsburgh, Pittsburgh, Pennsylvania, USA. ${ }^{3}$ Biostatistics Facility, University of Pittsburgh Cancer Institute, Pittsburgh, Pennsylvania, USA. ${ }^{4}$ Department of Radiology, University of Pittsburgh, Pittsburgh, Pennsylvania, USA. ${ }^{5}$ Department of Otolaryngology - Head and Neck Surgery, Oregon Health Sciences University, Portland, Oregon, USA. ${ }^{6}$ Department of Otolaryngology and ${ }^{7}$ Division of Medical Oncology University of Colorado, Denver, Colorado, USA. ${ }^{8}$ Department of Otolaryngology, Vanderbilt University, Nashville, Tennessee, USA. ${ }^{9}$ Department of Thoracic/Head and Neck Medical Oncology, MD Anderson Cancer Center, Houston, Texas, USA. ${ }^{10}$ Department of Pathology, University of Pittsburgh, Pittsburgh, Pennsylvania, USA. "Department of Medicine, University of Colorado, Denver, Colorado, USA. ${ }^{12}$ Department of Systems Biology, MD Anderson Cancer Center, Houston, Texas, USA.

BACKGROUND. EGFR and Src family kinases are upregulated in head and neck squamous cell carcinoma (HNSCC). EGFR interacts with Src to activate STAT3 signaling, and dual EGFR-Src targeting is synergistic in HNSCC preclinical models. pSrc overexpression predicted resistance to the EGFR inhibitor, erlotinib, in a prior window trial. We conducted a 4-arm window trial to identify biomarkers associated with response to ECFR and/or Src inhibition.

METHODS. Patients with operable stage II-IVa HNSCC were randomized to 7-21 days of neoadjuvant erlotinib, the Src inhibitor dasatinib, the combination of both, or placebo. Paired tumor specimens were collected before and after treatment. Pharmacodynamic expression of EGFR and Src pathway components was evaluated by IHC of tissue microarrays and reverse-phase protein array of tissue lysates. Candidate biomarkers were assessed for correlation with change in tumor size.

RESULTS. From April 2009 to December 2012, 58 patients were randomized and 55 were treated. There was a significant decrease in tumor size in both erlotinib arms $(P=0.0014)$; however, no effect was seen with dasatinib alone $(P=0.24)$. High baseline pMAPK expression was associated with response to erlotinib $(P=0.03)$. High baseline PSTAT3 was associated with resistance to dasatinib $(P=0.099)$.

CONCLUSIONS. Brief exposure to erlotinib significantly decreased tumor size in operable HNSCC, with no additive effect from dasatinib. Baseline PMAPK expression warrants further study as a response biomarker for anti-EGFR therapy. Basal expression of pSTAT3 may be independent of Src, explain therapeutic resistance, and preclude development of dasatinib in biomarker-unselected cohorts.

Authorship note: J.E. Bauman and U. Duvvuri contributed equally to this work.

Conflict of interest: The authors have declared that no conflict of interest exists.

Submitted: August 30, 2016

Accepted: February 1, 2017

Published: March 23, 2017

Reference information:

JCI Insight. 2017;2(6):e90449. https:// doi.org/10.1172/jii.insight.90449.
TRIAL REGISTRATION. NCT00779389.

FUNDING. National Cancer Institute, American Cancer Society, Pennsylvania Department of Health, V Foundation for Cancer Research, Bristol-Myers Squibb, and Astellas Pharma.

\section{Introduction}

Head and neck squamous cell carcinoma (HNSCC) constituted 3.6\% of new cancer diagnoses in the United States in 2015 (1). Despite advances in surgery, radiation, and systemic therapy, the overall survival of patients with HNSCC has improved only marginally over the past 3 decades, particularly in those with HPV-negative disease. While 6 FDA-approved drugs are available for the treatment of HNSCC, only 4 
are commonly used in the curative-intent setting: the cytotoxic chemotherapies cisplatin, 5-fluorouracil, docetaxel and the anti-EGFR mAb, cetuximab. Barriers to the identification of effective new drugs in HNSCC include the paucity of oncogenic driver mutations predicting response to specific molecular targeting agents and the limited availability of tumor specimens before and after treatment to assess the in situ pharmacodynamics of response. The window-of-opportunity clinical trial aims to overcome the latter barrier. In this trial model, patients undergo treatment with an anticancer therapy during the window between diagnosis and definitive surgery in order to assess paired tumor specimens for target modulation (2-5).

EGFR is a proproliferative, proinvasive, antiapoptotic $170-\mathrm{kDa}$ transmembrane protein within the RTK family implicated as an oncogene in HNSCC. Activated EGFR initiates pleiotropic downstream signaling cascades, including Ras/Raf/MAPK, PI3K/Akt, STAT3, and Src family kinases (SFKs) (6). EGFR is overexpressed and/or amplified in approximately $90 \%$ of HPV-negative $\operatorname{HNSCC}(7,8)$, in which higher EGFR expression levels are correlated with advanced stage, decreased survival, and decreased sensitivity to radiation and chemotherapy (9-12). The recognition of EGFR as both oncogene and prognostic biomarker compelled the investigation of EGFR inhibitors, including mAbs blocking the extracellular binding domain and small molecules abrogating intracellular RTK activity, in HNSCC. Cetuximab, an IgG1 antiEGFR mAb, improved clinical outcomes in phase III trials when added to radiation or platinum-based chemotherapy $(13,14)$. Although EGFR was the first validated molecular target in HNSCC, absolute improvement in a reliable clinical endpoint is limited to $10 \%-20 \%$ of patients, suggesting intrinsic resistance to EGFR inhibition, despite overexpression of the purported target in the majority of patients (13-15). The lack of association between clinical benefit and EGFR expression level or gene amplification highlights the dearth of predictive biomarkers to guide patient selection, a major unmet need $(16,17)$. Of particular interest are biomarkers that simultaneously represent bona fide targets, including the nonreceptor SFKs targeted by the small-molecule inhibitor, dasatinib.

EGFR interacts with Src to activate oncogenic STAT3 signaling in HNSCC. Dual blockade of EGFR and Src is synergistic in HNSCC cell lines, abrogating STAT3 activation and subsequent HNSCC proliferation (18). We previously reported that Src mediates erlotinib resistance in HNSCC via ligand-independent activation of Met (19). We completed a phase I trial demonstrating the safety of combined treatment with cetuximab and dasatinib in solid tumors, including HNSCC (20), and the combination of erlotinib and dasatinib was associated with a high rate of disease control during phase I/II testing for patients with advanced lung cancer (21). Finally, we identified high baseline phosphorylated Src (pSrc) expression as a resistance biomarker for erlotinib in a randomized window trial in patients with operable HNSCC (3). Although dasatinib has demonstrated negligible monotherapeutic activity in recurrent/metastatic HNSCC (22), the role of Src in the upstream transactivation of EGFR, parallel activation of compensatory RTK pathways, and downstream STAT3 signaling provide mechanistic support for dual EGFR-Src targeting. We conducted a randomized, double-blind window trial of erlotinib, dasatinib, the combination of both, or placebo control in patients with operable HNSCC, with the primary objective of identifying predictive biomarkers for EGFR and/or Src targeting that may translate to efficacy trials and the secondary objective of comparing the effect of biomarker modulation on tumor response.

\section{Results}

Subjects were well-balanced across treatment arms. From April 2009 through December 2012, 58 patients were randomized and 55 patients were treated across 7 centers. Patient allocation is presented in the CONSORT diagram (Figure 1). Baseline characteristics for the 55 treated subjects are summarized in Table 1. Randomization balance was assessed by exact $\chi^{2}$ tests of independence. Subjects were well-balanced among groups with respect to gender, anatomic site, primary tumor verses recurrence, and HPV status. A randomization imbalance was observed for American Joint Committee on Cancer (AJCC) stage, with a greater-than-expected frequency of stage I-II disease in the dasatinib arms and of stage III-IV disease in the erlotinib-only and placebo arms (exact $\chi^{2}, P=0.0097$ ). However, baseline tumor size (the sum of Response Evaluation Criteria in Solid Tumors-measurable [RECIST-measurable] index lesions) was balanced (Kruskal-Wallis; $P=0.58$ ). All 7 oropharynx tumors were 16 positive and 48 patients had nonoropharyngeal tumors; thus, $13 \%$ of the study cohort was classified as HPV positive and $87 \%$ as HPV negative.

Brief exposure to targeted therapy was well tolerated in the preoperative setting. Brief exposure to erlotinib, dasatinib, the combination of both, or placebo was well tolerated in the preoperative setting. Treatmentemergent adverse events are summarized in Table 2, irrespective of attribution. Acneiform rash and 


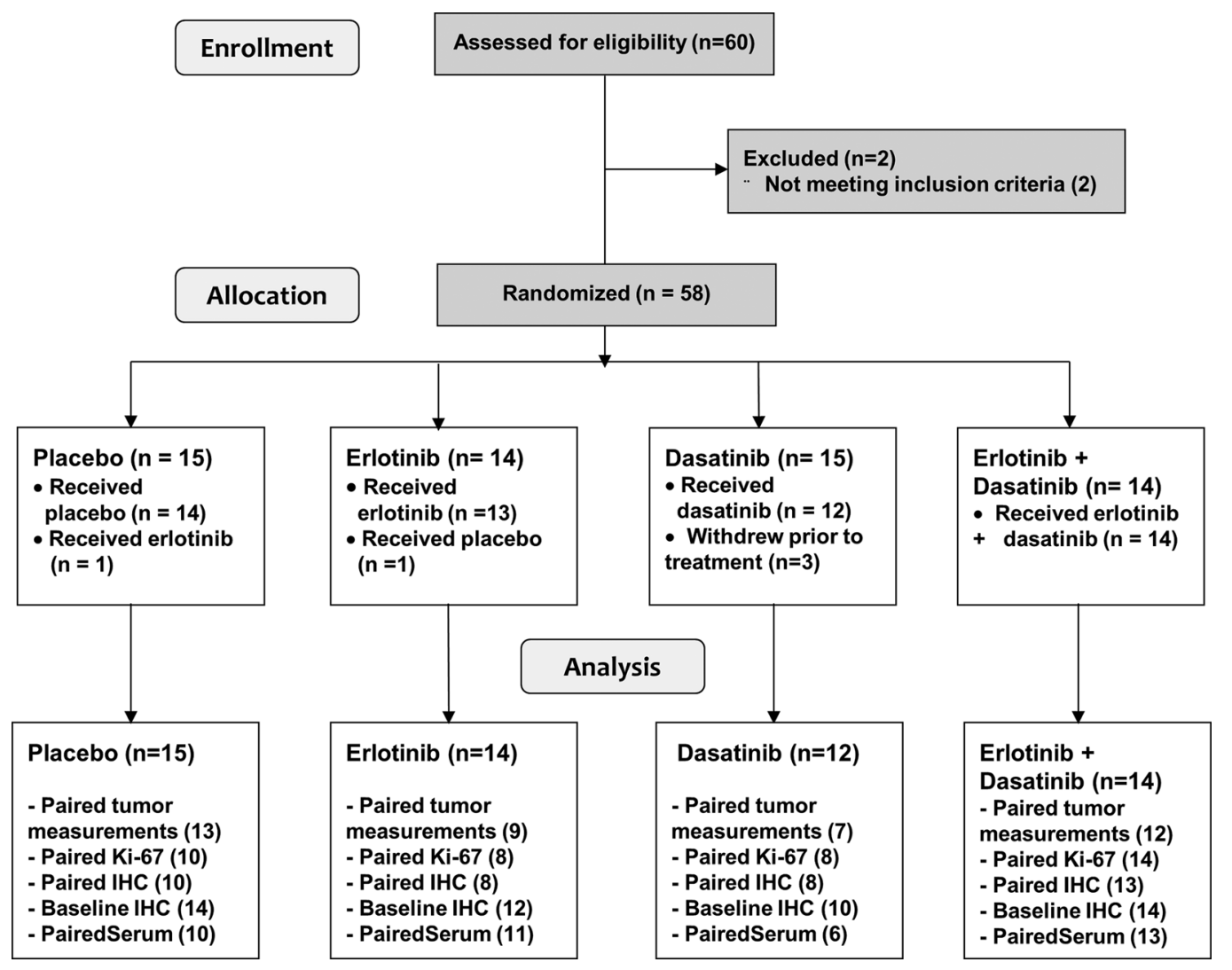

Figure 1. CONSORT diagram. Patient enrollment, allocation, and biospecimen analysis are specified according to treatment arm.

diarrhea were observed more often in the erlotinib arms, as expected for small-molecule EGFR inhibitors (23); however, no other safety differences were seen. No treatment-related or perioperative deaths were observed. The rate of serious postoperative complications was low and in line with clinical experience, as detailed by arm in Table 2.

Preoperative erlotinib, alone or in combination with dasatinib, significantly decreased HNSCC tumor size. Single-agent EGFR inhibitors are associated with RECIST response rates of only $4 \%-13 \%$ in recurrent/ metastatic HNSCC $(24,25)$ and these responses typically emerge following 2-4 months of continuous treatment. In the current study, given the brief duration of exposure, categorical RECIST responses would be uninformative. Instead, we used well-characterized RECIST measurement techniques to establish the sum of index lesions at baseline and after treatment and then used the proportional change in tumor size as the primary endpoint. Tumor size before treatment was equally distributed across treatment arms $(P=$ 0.582). A waterfall plot for the 41 patients with paired, RECIST-measurable index lesions is displayed in Figure 2A. As shown in Figure 2B, erlotinib, either alone or in combination with dasatinib, resulted in a significant reduction in tumor size compared with dasatinib alone or placebo $(P=0.0006)$. Tests for main effect $(P=0.29)$ and interaction $(P=0.49)$ confirmed no significant additive or detrimental effect from dasatinib. Patients were treated for a median of 14 days (range 2-21 days), with treatment times differing by treatment $\operatorname{arm}(P=0.0305)$; median treatment duration was 15 and 16 days for erlotinib and placebo, respectively, compared with the 14 days for dasatinib or the combination. Notably, longer treatment duration within the 7 to 21 day range did not enhance the observed antitumor effect of erlotinib $(P=0.80)$.

Short-term exposure to EGFR and SFK-targeting agents had no effect on biomarker modulation, as measured by IHC. We measured 14 proteins by IHC (pSTAT1, pSTAT3, pAKT, pSRC, pMAPK, pEGFR, pMET, pFAK, cMet, EGFR, vimentin, Ecadherin, HER2, and HER3), as well as 2 serum analytes (IL-6 and TGF- $\alpha$ ) at baseline and again just prior to surgery to assess biomarker modulation over the 2-week treatment period. We tested whether change in value of these 16 biomarkers was associated with treatment arm with 2-way ANOVA. Neither erlotinib nor dasatinib, alone or in combination, significantly altered any of the 16 biomarkers as compared with placebo (Supplemental Figure 1; supplemental material available online with this article; https://doi.org/10.1172/jci.insight.90449DS1). 
Table 1. Baseline patient characteristics

\begin{tabular}{|c|c|c|c|c|c|c|}
\hline Characteristic & Total (100\%) & Placebo & Erlotinib & Dasatinib & Erlotinib + dasatinib & Test of equality ${ }^{A}$ \\
\hline$n(\%)$ & 55 & 15 & 14 & 12 & 14 & $P$ value \\
\hline Gender & & & & & & 0.6221 \\
\hline Age (yr) & & & & & & 0.0365 \\
\hline Median (yr) & 61 & 63 & 52 & 64 & 62 & \\
\hline IQR & $51-66$ & $58-66$ & $49-61$ & $51-71$ & $59-66$ & \\
\hline Oropharynx & $7(13)$ & $2(13)$ & $3(21)$ & $0(0)$ & $2(14)$ & \\
\hline Larynx & $6(11)$ & $0(0)$ & $1(7)$ & $2(17)$ & $3(21)$ & \\
\hline ECOG performance status & & & & & & 0.8301 \\
\hline 0 & $29(53)$ & $8(53)$ & $8(47)$ & $5(42)$ & $8(57)$ & \\
\hline 1 & $25(45)$ & $7(47)$ & $6(43)$ & $6(50)$ & $6(43)$ & \\
\hline III & $11(20)$ & $4(27)$ & $2(14)$ & $0(0)$ & $5(36)$ & \\
\hline IV & $28(50)$ & $4(27)$ & $5(36)$ & $10(83)$ & $9(64)$ & \\
\hline Treatment site & & & & & & 0.0606 \\
\hline Colorado & $3(5)$ & $0(0)$ & $2(14)$ & $1(8)$ & $0(0)$ & \\
\hline MD Anderson & $2(4)$ & $0(0)$ & $0(0)$ & $2(17)$ & $0(0)$ & \\
\hline Oregon & $10(18)$ & $4(27)$ & $2(14)$ & $1(8)$ & $3(21)$ & \\
\hline Oregon VA & $1(2)$ & $0(0)$ & $1(7)$ & $0(0)$ & $0(0)$ & \\
\hline Pittsburgh & $30(55)$ & $9(60)$ & $8(57)$ & $3(25)$ & $10(71)$ & \\
\hline Pittsburgh VA & $1(2)$ & $0(0)$ & $0(0)$ & $1(8)$ & $0(0)$ & \\
\hline Vanderbilt & $8(15)$ & $2(13)$ & $1(7)$ & $4(33)$ & $1(7)$ & \\
\hline Tumor type & & & & & & 0.7697 \\
\hline
\end{tabular}

${ }^{A} \chi^{2}$ test for categorical data; Kruskal-Wallis test for continuous variables. AJCC, American Joint Committee on Cancer; ECOC, Eastern Cooperative Oncology Group; IQR, interquartile range.

Baseline signaling biomarkers may predict tyrosine kinase inhibitor sensitivity or resistance. In the absence of oncogenic driver mutations, the identification of tumor biomarkers that predict response to targeted therapy has been a major challenge in HNSCC. Activating mutations in the EGFR kinase domain, associated with erlotinib response in lung cancer, are extremely rare in $\operatorname{HNSCC}(26,27)$. Furthermore, neither EGFR expression nor gene copy number predicts cetuximab response $(16,17)$. Based on an understanding of EGFR and Src biology in HNSCC, we prioritized 4 phosphorylated proteins in the baseline tumor (pSTAT3, pSrc, pMet, and pMAPK) as well as baseline serum IL-6 and assessed whether their expression was associated with radiologic response to treatment. Tissue of sufficient quality to assess baseline tumor biomarkers by IHC of the tissue microarrays was available in 50 of 55 patients (91\%). As shown in Figure 3A, baseline pMAPK expression was associated with erlotinib sensitivity $(P$ $=0.099)$. One patient experienced an exceptional, near-complete histologic response of an oral cavity primary tumor to single-agent erlotinib, which we found to be associated with a MAPK1E322K mutation (28). Identifying biomarkers of resistance is also critical to guide the use of targeted therapies. In this study, baseline pSTAT3 expression was associated with dasatinib resistance ( $P=0.0223$; Figure $3 \mathrm{~B})$. Baseline tumor expression of pSrc or pMet, or serum level of IL-6, was not associated with treatment response (data not shown).

Erlotinib and dasatinib significantly modulated their respective signaling targets by reverse-phase protein array. Erlotinib is an orally active quinazoline derivative that reversibly blocks ATP binding in the tyrosine kinase domain of EGFR, thereby inhibiting autophosphorylation. EGFR is autophosphorylated on 5 

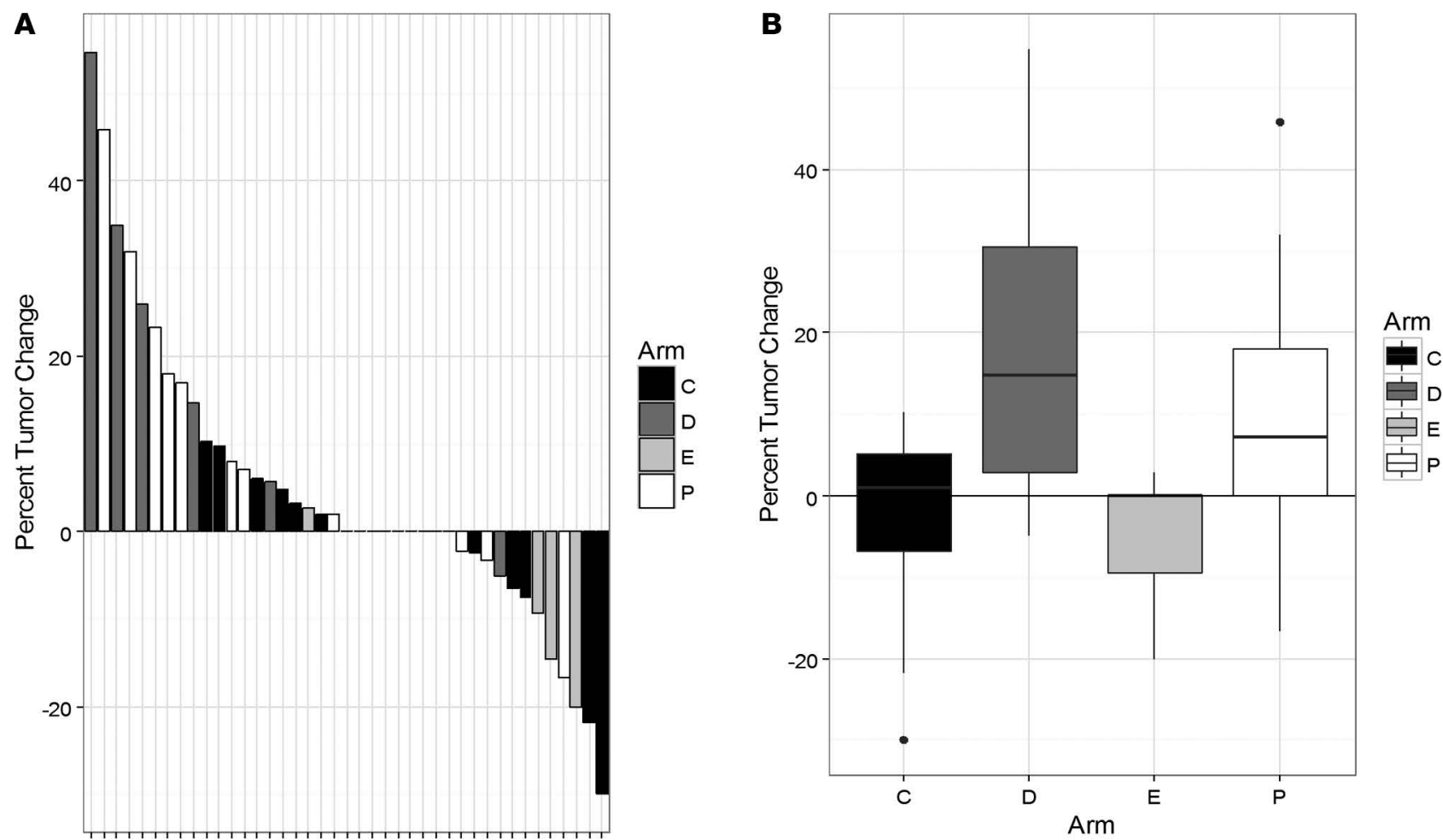

Figure 2. Response by treatment arm. (A) Waterfall plot for individual patient responses, as measured by percentage change in RECIST-measurable index lesions, presented by treatment arm. Each box represents one of the 41 patients with CT scan data both before and after treatment. (B) Box-and-whisker plots showing the distribution of percentage change in tumor size for each of the 4 treatment arms. Boxes show the interquartile range, with the median shown as a dark horizontal line; whiskers extend to 1.5 times the interquartile range. Percentage change in RECIST-measurable index lesions was compared among groups by 2-way ANOVA. Erlotinib was associated with reduction in tumor size $(P=0.0006)$. Tests for main effect $(P=0.29)$ and interaction $(P=0.49)$ confirmed no significant favorable or detrimental effect from dasatinib. C, combination; D, dasatinib; E, erlotinib; P, placebo.

cytoplasmic tyrosine residues. Assessment of each autophosphorylation site is limited by the quality of available antisera, particularly for IHC analyses. As measured by immunoblotting, expression of pEGFR on a nonautophosphorylation site, pY845, was reportedly decreased in 7 HNSCC patients treated with 7 days of erlotinib on a window trial (29). Curiously, pY845 has been previously associated with Src signaling (30). In this study, we quantified pEGFR and pSrc expression in paired, fresh-frozen tumors by reverse-phase protein array (RPPA); 39 of 55 (71\%) patients provided specimens of sufficient quality for this analysis. Among the 4 EGFR phosphorylation sites tested, the pY1068 autophosphorylation site was decreased in the erlotinib versus nonerlotinib arms $(P=0.0444$, adjusted $P=0.18)$, while among 5 Src phosphorylation sites, pY527 was decreased in the dasatinib versus nondasatinib arms $(P=0.0455$, adjusted $P=0.23)$. In contrast, neither $\mathrm{pEGFR}$ nor $\mathrm{pSrc}$ expression by IHC significantly decreased during erlotinib or dasatinib treatment, which may reflect the higher sensitivity and specificity of RPPA for selected phosphorylation sites. Collectively, these findings suggest that both erlotinib and dasatinib hit their respective targets. However, while both were molecularly active in unselected patients with operable HNSCC, only erlotinib resulted in clinically detectable activity.

Change in the Ki67 proliferative index is not associated with clinical response by CT scan. Window trials can determine the pharmacodynamic effect of an agent within a tumor. However, the clinical relevance of pharmacodynamic changes relies upon their relationship to a surrogate histologic measure, such as the Ki67 proliferation index, or clinical measure, such as serial radiographic imaging. We previously reported that erlotinib significantly decreased Ki67 labeling after 7 to 14 days of treatment on a window trial (3). However, imaging after treatment was not obtained in that study, limiting clinical interpretation of the finding. In the present trial, we obtained paired intrapatient measurements of both Ki67 and tumor size. Unexpectedly, change in Ki67 did not correlate with change in tumor size $\left(\mathrm{R}^{2}=0.10 ; P=\right.$ 0.23 ), suggesting that change in tumor size is the preferred surrogate marker in HNSCC window trials. 
A

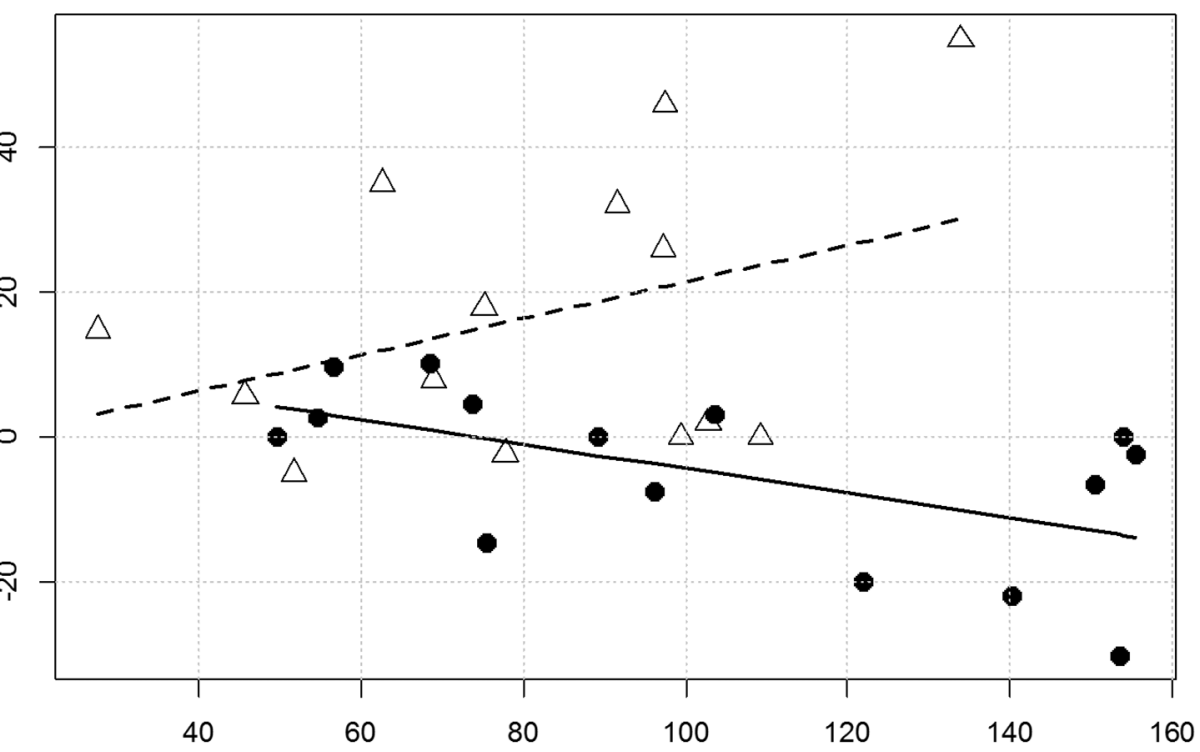

-- Erlotinib

$\triangle-$ No Erlotinib

Baseline pMAPK

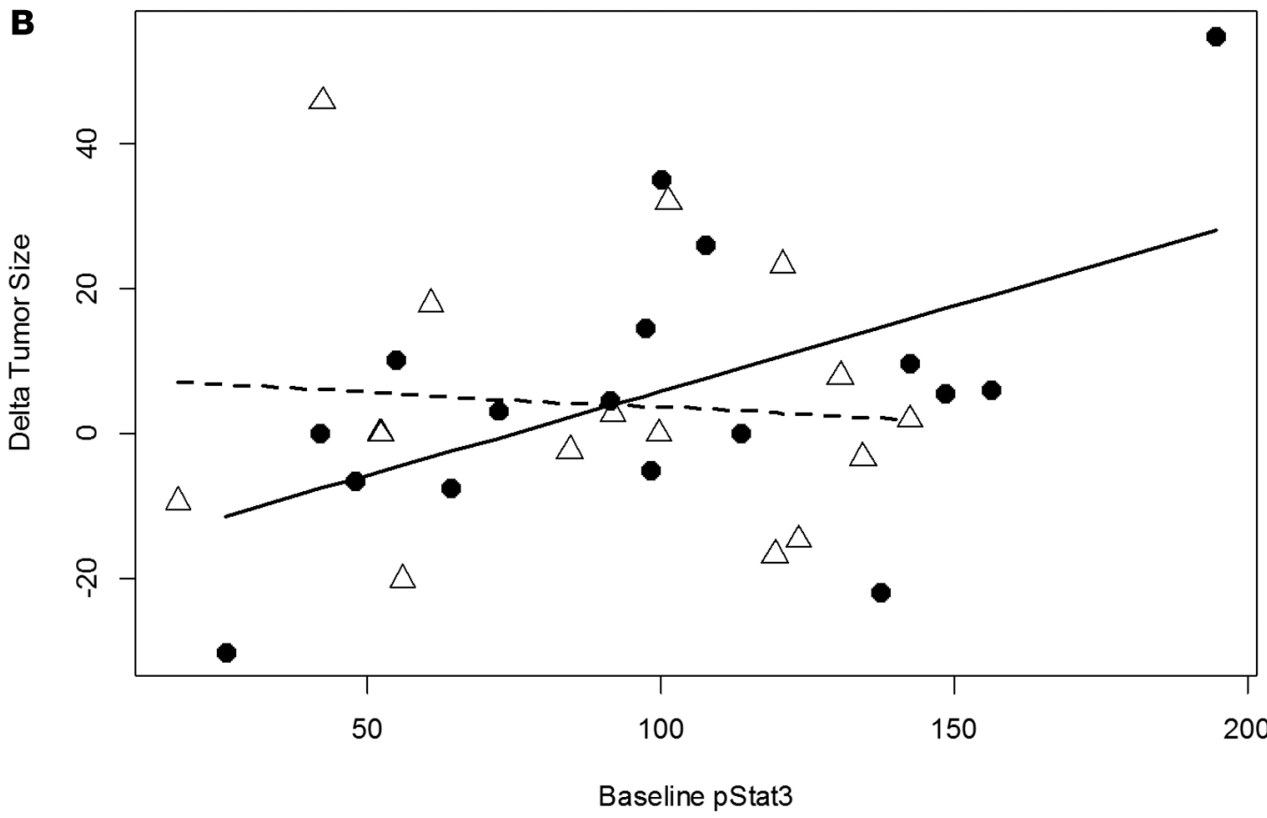

- Dasatinib

- No Dasatinib

Figure 3. Baseline IHC biomarkers of response or resistance. (A) Among erlotinib-treated patients, baseline pMAPK was inversely associated with response. An analysis of covariance found the presence of interaction between baseline pMAPK and erlotinib exposure $(P=0.0232)$. Separate slope estimates were calculated: the slope for patients not exposed to erlotinib was positive $(0.25, P=0.092)$, whereas the slope for the erlotinib group was negative (-0.169, $P=0.099)$, suggesting that greater baseline pMAPK protein levels enhanced erlotinib antitumor response. (B) Among dasatinib-treated patients, baseline PSTAT3 was associated with resistance. An analysis of covariance suggests moderate interaction between baseline pStat 3 and dasatinib exposure $(P=0.0875)$. Separate slope estimates were calculated: the slope for the no dasatinib group was not different from $0(P=0.7334)$, whereas the slope for the dasatinib group was positive $(P=0.0235)$, suggesting that greater baseline pStat3 protein levels contributed to dasatinib resistance.

\section{Discussion}

EGFR is a validated therapeutic target in HNSCC. However, unlike lung cancer, in which EGFR driver mutations predict response to erlotinib, or colorectal cancer, in which activating $K R A S$ and $B R A F$ mutations predict resistance to cetuximab (31), no predictive genomic biomarkers exist in HNSCC $(16,17)$. Moreover, neither EGFR expression nor gene amplification is associated with response to EGFR inhibitors in HNSCC $(16,17)$. Instead, aberrantly activated signaling proteins within the EGFR network have been 
Table 2. Treatment-emergent adverse events and perioperative complications

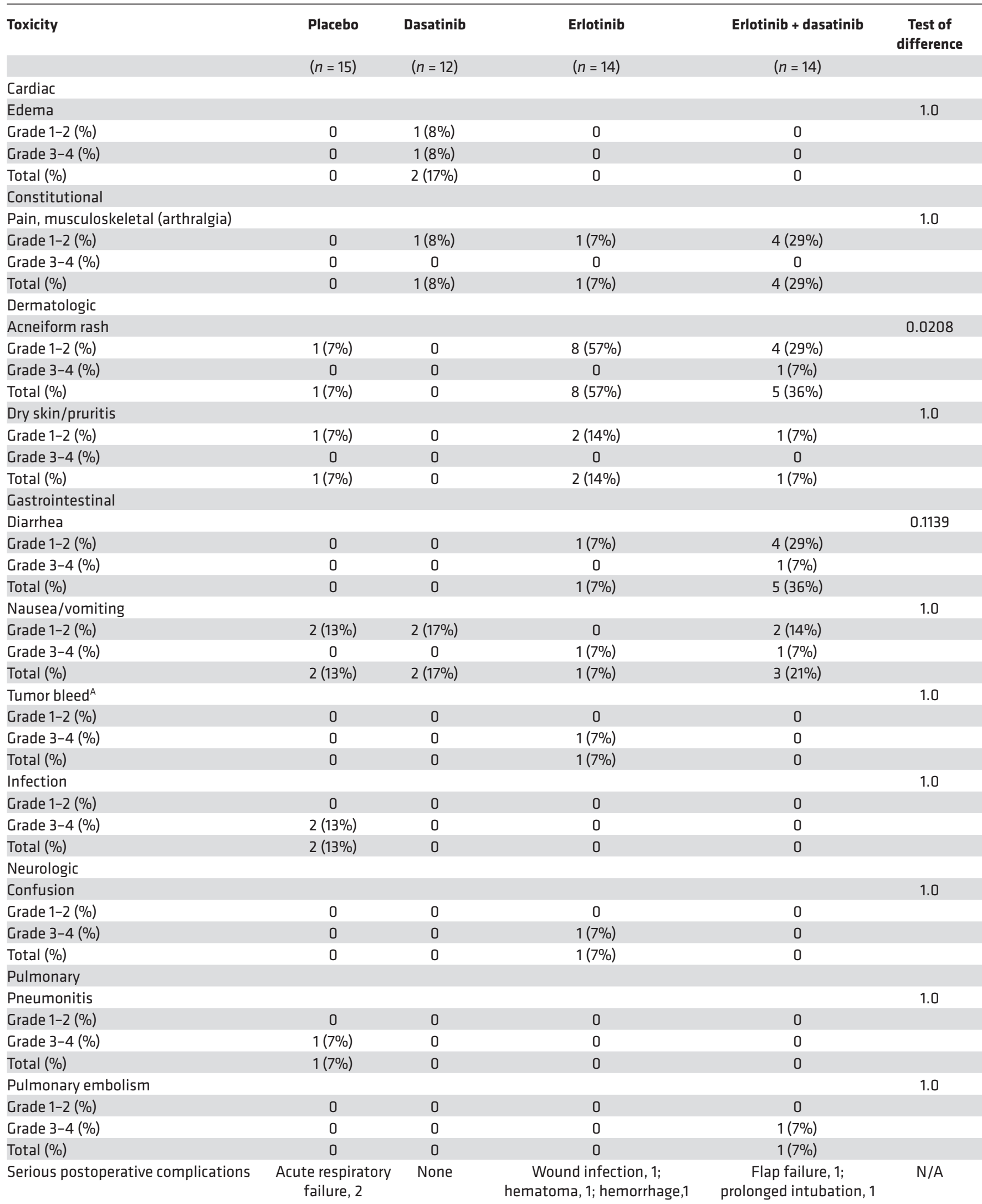

${ }^{\text {AC }}$ rade 3 tumor bleed was considered unrelated to the study drug; during bleeding, the study drug was held and then restarted after successful cautery. No bleeding recurrence was observed with rechallenge. 
investigated. Candidate protein biomarkers that both predict resistance to EGFR inhibition and represent drug targets themselves are of particular clinical interest.

SFKs are critical for EGFR-dependent and -independent signaling in $\operatorname{HNSCC}(18,32,33)$ and are targetable by the broad-spectrum SFK inhibitor, dasatinib. Notably, Src is activated both as a consequence of EGFR signaling and as a precursor to EGFR signaling when G-coupled protein receptors recruit pSrc to the upstream complex mediating EGFR transactivation $(18,32,34)$. In preclinical HNSCC models, activation of Src causes resistance to EGFR inhibition $(19,35,36)$. In patients with HNSCC, elevated baseline pSrc expression was associated with resistance to the antiproliferative effects of erlotinib during our first window trial (37). Based upon these mechanistic and clinical insights, we hypothesized that combined EGFR-Src targeting would circumvent an important resistance mechanism to erlotinib and result in heightened clinical activity. In the present randomized, placebo-controlled window trial, erlotinib significantly decreased tumor size in patients with operable HNSCC, in line with the cumulative literature regarding EGFR targeting. However, despite RPPA evidence that both erlotinib and dasatinib inhibited their respective targets, dual EGFR-Src inhibition resulted in no additive or synergistic clinical activity.

MAPKs are activated by numerous upstream stimuli, including nonreceptor kinases and RTKs, such as EGFR and Src. In this trial, baseline pMAPK expression was associated with erlotinib response, irrespective of the presence of dasatinib. Moreover, one patient who demonstrated an exceptional response to erlotinib harbored an activating $M A P K 1^{E 322 K}$ mutation (28). Collectively, these findings suggest that basal MAPK activation, as established by IHC or whole exome sequencing, indicates EGFR pathway dependence and represents a predictive biomarker for the EGFR tyrosine kinase inhibitor. Activation of Janus kinase via an IL-6 feedback loop, which bypasses phosphorylation of STAT3 by SFKs, is an established mechanism of acquired resistance to dasatinib (38). Consistent with these preclinical data, we observed that baseline overexpression of pSTAT3 was associated with tumor progression during dasatinib treatment, suggesting that SFK-independent activation of STAT3 is a de novo mechanism of resistance to dasatinib in patients with HNSCC.

The window trial has been successfully deployed in HNSCC to mechanistically dissect the molecular activity of single-agent erlotinib (2), erlotinib versus erlotinib-sulindac (3), lapatinib (4), an oligonucleotide STAT3 decoy (5), and single-agent cetuximab (NCT01218048). However, interpreting the clinical relevance of pharmacodynamic changes requires a relationship to a surrogate measure of clinical activity. In our previous window trial, we reported that erlotinib significantly decreased Ki67 labeling after 7 to 14 days of treatment and this effect was potentiated by the cyclooxygenase inhibitor, sulindac (3). Although change in Ki67 is a validated surrogate biomarker in neoadjuvant studies investigating targeted therapy in breast cancer (39-41), Ki67 modulation has not been evaluated directly against clinical outcome in HNSCC (2, 4, 5). To address this knowledge gap, we conducted both measurements in this study and found that change in the Ki67 proliferation index was unrelated to change in tumor size, an accepted metric of clinical activity. Notably, RECIST responses are rarely observed in window trials, in which the duration of therapy is short and nontherapeutic. However, tumor regression is frequently seen and can be quantified $(2,4)$. For example, among 31 patients treated with preoperative erlotinib for a median of 20 days, $29 \%$ demonstrated measurable regression of HNSCC tumors (2). In this trial, with a median of 14 days of treatment (interquartile range $=14-17$ days), measurable tumor regression was observed in 12 of $41(29 \%)$ patients with paired RECIST measurements, while regression of $10 \%$ or more was observed in $5(12 \%)$. In the design of future HNSCC window trials, we recommend a minimum of 2 weeks of treatment and adapted, quantitative RECIST criteria, as presented here, to separate responders from nonresponders.

In summary, EGFR targeting with erlotinib significantly reduced tumor size in patients with operable HNSCC, when administered alone or in combination with dasatinib compared with dasatinib alone or placebo. Baseline hyper-activation of MAPK, as assessed by IHC in the overall trial population or by the presence of a rare $M A P K 1^{E 322 K}$ mutation in a single patient, was associated with response to erlotinib and suggests pathway dependence. Both pMAPK expression and genomic MAPK activation warrant further study as predictive biomarkers for EGFR inhibitors in HNSCC. Baseline SFK-independent activation of the oncogene STAT3 may explain the failure of dasatinib to enhance erlotinib's clinical activity. Efficacy studies evaluating dual EGFR-Src targeting cannot be justified in biomarker-unselected patients with HNSCC; however, they remain of interest in patients with low basal STAT3 activation. The combination of cetuximab-dasatinib is now under investigation in patients with recurrent/metastatic HNSCC selected for low serum IL-6 levels, an accessible surrogate for low JAK/STAT3 activity (NCT01488318) (42). 


\section{Methods}

Trial design. This was a randomized, 4-arm, parallel group window trial in patients with operable HNSCC scheduled for curative-intent surgery, designed to evaluate differential biomarker modulation in paired tumor specimens by 1 of 3 treatments, as compared with placebo. Subjects were allocated on a 1:1:1:1 ratio to 1 of 4 treatment arms, using a computerized random number generator operated by the University of Pittsburgh Biostatistics Facility. Treatment with erlotinib or placebo (for erlotinib) was double blind. Assignment to dasatinib was open label, as there was no placebo for dasatinib.

Eligibility criteria. Eligible subjects met the following key inclusion criteria: stage II-IVa, histologically confirmed HNSCC of the oral cavity, oropharynx, hypopharynx, or larynx as defined by the AJCC Staging Handbook, 7th edition (43); planned primary or salvage resection of the primary tumor; age $\geq 18$ years; ECOG performance status 0-2; and adequate hematologic, hepatic, and renal function. Key exclusion criteria included prior treatment with an EGFR inhibitor; any grade pleural or pericardial effusion; major medical comorbidities, including class III-IV congestive heart failure, ventricular arrhythmia, prolonged QTc; or bleeding disorder that may increase the risk of targeted therapy or surgery.

Treatment plan. The 4 treatment groups included placebo (for erlotinib), erlotinib (150 mg daily), placebo plus dasatinib (100 mg daily), or erlotinib (150 mg daily) plus dasatinib (100 mg daily). Preoperative therapy was administered for 7 to 21 days, with the length of treatment depending upon the planned date of surgery, and was discontinued 24-36 hours before surgery. If surgery was delayed for logistical reasons, study treatment was continued until 1 day prior to surgery, for a maximum of 28 days. Compliance was monitored with daily drug diaries. Toxicities were described according to National Cancer Institute Common Terminology Criteria for Adverse Events, version 4.0. Subjects underwent CT scans within 4 weeks of initiating study treatment and then within 72 hours prior to surgery. All tumor measurements before and after treatment were performed according to RECIST version 1.1 (44) by a single neuroradiologist blinded to treatment assignment.

Biospecimen collection and analysis. Tumor specimens before and after treatment were obtained at the time of diagnostic evaluation and definitive resection. A representative portion of each tumor (the equivalent of at least two 4-mm biopsies or two 18-gauge core needle biopsies) was snap frozen in liquid nitrogen and stored at $-80^{\circ} \mathrm{C}$. A similar-sized sample was formalin fixed and paraffin embedded. Specimens were shipped to the University of Pittsburgh for centralized analyses. The primary method for analyzing tumor biomarkers was IHC analysis of tissue microarrays as previously described (3). Antibodies and specifications, including phosphorylation site when applicable, are summarized in Supplemental Table 1. Tumor protein expression was scored quantitatively with Aperio computerassisted digital analysis by a research pathologist, who was blinded to patient identity and treatment assignment. Ki67 was scored according to international consensus guidelines (45). HPV status of oropharyngeal tumors was determined by p16 IHC according to standard clinical practice (46). RPPA, a high-throughput quantitative proteomics platform that interrogates $\geq 200$ proteins, including a majority of the cancer-related signaling pathways, was performed on lysates from snap-frozen specimens as previously described (47).

Serum was isolated according to standard techniques and immediately aliquoted, frozen, and stored at $-80^{\circ} \mathrm{C}$. An ELISA (Quantikine ELISA kits, R\&D Systems) was used to quantify circulating IL-6.

Statistics. The primary objective of this window trial was to determine if brief preoperative exposure to erlotinib, dasatinib, or the combination of both results in differential biomarker response in patients with operable HNSCC, as measured by change in EGFR and Src pathway-related proteins during the preoperative window. The secondary objective was to measure the percentage of change in the sum of RECIST-measurable index lesions on CT scans before and after treatment and to establish whether tumor response was associated with biomarker expression. Differences among treatment groups were analyzed by 2-way ANOVA, including tests for interaction between erlotinib and dasatinib; a main effect or interaction was considered significant at $\alpha=0.05$. Finally, we aimed to identify baseline biomarkers that were associated with CT response as well as to confirm modulation of the respective targets of erlotinib (pEGFR) and dasatinib (pSrc). The recommended sample size was based upon the primary objective: the detection of group differences in biomarker modulation. The calculated sample size of 56 patients (14/arm) provided $80 \%$ power to detect a $1-1$ og difference in treatment effect between any 2 groups with a 2 -tailed Wilcoxon test at $\alpha=0.01$. The protocol specified that biomarkers were to reflect modulation of the EGFR/Src pathways. To this end, biomarker 
analysis proceeded according to two predefined tiers. Five baseline analytes that were mechanistically associated with preclinical sensitivity to erlotinib and/or dasatinib were tested for association between change in tumor size and baseline expression using linear regression with a shared $\alpha$ of 0.15 : tumor pSTAT3, pSrc, pMet, or pMAPK and serum IL-6. For these prioritized biomarkers, a $P$ value of less than or equal to 0.03 was considered significant. For the remaining 11 candidate proteins, multiple testing was corrected for false discovery by the Benjamini and Hochberg method and reported as positive if expected false discovery was $\leq 0.10$ (48). An analysis of covariance was conducted to assess whether any baseline effect of pMAPK differed by exposure to erlotinib. An analogous procedure was applied to baseline pSTAT3 and dasatinib. The analysis of biomarker modulation by treatment group proceeded with a 2-way ANOVA with interaction. RPPA was employed as an unsupervised proteomic approach to biomarker discovery. As a secondary objective, we assessed the correlation between CT response and change in the Ki67 proliferation index, as used in our prior window study (3), to assess whether change in Ki67 is a valid surrogate for clinical activity in HNSCC window trials. We also assessed the effect of baseline biomarker levels on $\mathrm{CT}$ response by linear regression. Frequencies of total toxicities by toxicity class were tested for treatment arm differences by Fisher's exact test with a Bonferroni correction. All tests were 2 tailed.

Study approval. This multicenter trial was approved by the institutional review boards of the University of Pittsburgh, the Pittsburgh Veterans Affairs Medical Center (VAMC), Oregon Health \& ScienceUniversity, the Portland VAMC, Vanderbilt University, the University of Colorado Health Center, and the MD Anderson Cancer Center. The trial was nationally registered at clinicaltrials.gov (NCT00779389). All subjects provided written, informed consent.

\section{Author contributions}

Conception and design were by JEB, WEG, and JRG. Acquisition of data was performed by JEB, UD, NDG, JS, AJ, WGY, FMJ, JTJ, RLF, SK, FRH, KE, MS, and JRG. Analysis and interpretation of data were performed by JEB, UD, WEG, TJR, LW, SC, RLF, GBM, MS, and JRG. Writing, review, and/or revision of the manuscript were performed by all authors. Administrative, technical, or material support was provided by JEB, UD, JK, KE, JTF, GBM, and JRG. Blinded RECIST analysis was performed by TJR. Aperio computer-assisted digital analysis was performed by LW.

\section{Acknowledgments}

This work was supported by grants from the National Cancer Institute (P50CA097190 to JRG and RLF; the University of Pittsburgh Cancer Institute Biostatistics Core Facility was supported in part by P30CA047904), the American Cancer Society (CRP-08-229-01 to JRG), and the Pennsylvania Department of Health. Support was also provided by the Investigator-Sponsored Trial programs of BristolMyers Squibb and Astellas Pharma. JEB was supported in part by the V Foundation for Cancer Research. UD was supported in part by funds from the Department of Veterans Affairs Biomedical Laboratory Research and Development. This work does not reflect the views of the Pennsylvania state government, the United States government, or the Department of Veterans Affairs.

Address correspondence to: Jennifer R. Grandis, 550 16th Street, 6th floor, San Francisco, California 94143, USA. Phone: 415.514.8084; E-mail: Jennifer.Grandis@ucsf.edu.

JEB's present address is: University of Arizona, Tucson, Arizona, USA.

NDG's present address is: MD Anderson Cancer Center, Houston, Texas, USA.

WGY's present address is: Yale University, New Haven, Connecticut, USA.

JK's present address is: Boston University, Boston, Massachusetts, USA.

JRG's present address is: UCSF, San Francisco, USA. 
1. Siegel RL, Miller KD, Jemal A. Cancer statistics, 2015. CA Cancer J Clin. 2015;65(1):5-29.

2. Thomas F, et al. Pilot study of neoadjuvant treatment with erlotinib in nonmetastatic head and neck squamous cell carcinoma Clin Cancer Res. 2007;13(23):7086-7092.

3. Gross ND, et al. Erlotinib, erlotinib-sulindac versus placebo: a randomized, double-blind, placebo-controlled window trial in operable head and neck cancer. Clin Cancer Res. 2014;20(12):3289-3298.

4. Del Campo JM, et al. Effects of lapatinib monotherapy: results of a randomised phase II study in therapy-naive patients with locally advanced squamous cell carcinoma of the head and neck. Br J Cancer. 2011;105(5):618-627.

5. Sen M, et al. First-in-human trial of a STAT3 decoy oligonucleotide in head and neck tumors: implications for cancer therapy. Cancer Discov. 2012;2(8):694-705.

6. Ciardiello F, Tortora G. Epidermal growth factor receptor (EGFR) as a target in cancer therapy: understanding the role of receptor expression and other molecular determinants that could influence the response to anti-EGFR drugs. Eur J Cancer. 2003;39(10):1348-1354.

7. Rubin Grandis J, et al. Levels of TGF-alpha and EGFR protein in head and neck squamous cell carcinoma and patient survival. J Natl Cancer Inst. 1998;90(11):824-832.

8. Chung $\mathrm{CH}$, et al. Increased epidermal growth factor receptor gene copy number is associated with poor prognosis in head and neck squamous cell carcinomas. J Clin Oncol. 2006;24(25):4170-4176.

9. Ang KK, et al. Impact of epidermal growth factor receptor expression on survival and pattern of relapse in patients with advanced head and neck carcinoma. Cancer Res. 2002;62(24):7350-7356.

10. Etienne MC, et al. A multifactorial approach including tumoural epidermal growth factor receptor, p53, thymidylate synthase and dihydropyrimidine dehydrogenase to predict treatment outcome in head and neck cancer patients receiving 5-fluorouracil. Br J Cancer. 1999;79(11-12):1864-1869

11. Numico G, et al. EGFR status and prognosis of patients with locally advanced head and neck cancer treated with chemoradiotherapy. Anticancer Research. 2010;30(2): 671-676.

12. Gupta AK, et al. Local recurrence in head and neck cancer: relationship to radiation resistance and signal transduction. Clin Cancer Res. 2002;8(3):885-892.

13. Vermorken JB, et al. Platinum-based chemotherapy plus cetuximab in head and neck cancer. N Engl J Med. 2008;359(11):1116-1127.

14. Bonner JA, et al. Radiotherapy plus cetuximab for squamous-cell carcinoma of the head and neck. NEngl J Med. 2006;354(6):567-578.

15. Vermorken JB, Herbst RS, Leon X, Amellal N, Baselga J. Overview of the efficacy of cetuximab in recurrent and/or metastatic squamous cell carcinoma of the head and neck in patients who previously failed platinum-based therapies. Cancer. 2008;112(12):2710-2719.

16. Licitra L, et al. Evaluation of EGFR gene copy number as a predictive biomarker for the efficacy of cetuximab in combination with chemotherapy in the first-line treatment of recurrent and/or metastatic squamous cell carcinoma of the head and neck: EXTREME study. Ann Oncol. 2011; 22 (5): 1078-1087.

17. Licitra L, et al. Predictive value of epidermal growth factor receptor expression for first-line chemotherapy plus cetuximab in patients with head and neck and colorectal cancer: analysis of data from the EXTREME and CRYSTAL studies. Eur J Cancer. 2013;49(6):1161-1168.

18. Xi S, et al. Src kinases mediate STAT growth pathways in squamous cell carcinoma of the head and neck. J Biol Chem. 2003;278(34):31574-31583.

19. Stabile LP, et al. c-Src activation mediates erlotinib resistance in head and neck cancer by stimulating c-Met. Clin Cancer Res. 2013;19(2):380-392.

20. Argiris A, et al. Phase I and pharmacokinetic study of dasatinib and cetuximab in patients with advanced solid malignancies. Invest New Drugs. 2012;30(4):1575-1584.

21. Haura EB, et al. Phase I/II study of the Src inhibitor dasatinib in combination with erlotinib in advanced non-small-cell lung cancer. J Clin Oncol. 2010;28(8):1387-1394.

22. Brooks HD, et al. Phase 2 study of dasatinib in the treatment of head and neck squamous cell carcinoma. Cancer. 2011;117(10):2112-2119.

23. Shepherd FA, et al. Erlotinib in previously treated non-small-cell lung cancer. N Engl J Med. 2005;353(2):123-132.

24. Perez CA, et al. Phase II study of gefitinib adaptive dose escalation to skin toxicity in recurrent or metastatic squamous cell carcinoma of the head neck. Oral Oncol. 2012:48(9);887-892.

25. Soulieres D, Senzer NN, Vokes EE, Hidalgo M, Agarwala SS, Siu LL. Multicenter phase II study of erlotinib, an oral epidermal growth factor receptor tyrosine kinase inhibitor, in patients with recurrent or metastatic squamous cell cancer of the head and neck. J Clin Oncol. 2004;22(1):77-85.

26. Stransky N, et al. The mutational landscape of head and neck squamous cell carcinoma. Science. 2011;333(6046):1157-1160

27. Agrawal N, et al. Exome sequencing of head and neck squamous cell carcinoma reveals inactivating mutations in NOTCH1. Science. 2011;333(6046):1154-1157.

28. Van Allen EM, et al. Genomic correlate of exceptional erlotinib response in head and neck squamous cell carcinoma. JAMA Oncol. 2015;1(2):238-244.

29. Tsien CI, et al. Effect of erlotinib on epidermal growth factor receptor and downstream signaling in oral cavity squamous cell carcinoma. Head Neck. 2013;35(9):1323-1330.

30. Boerner JL, Demory ML, Silva C, Parsons SJ. Phosphorylation of Y845 on the epidermal growth factor receptor mediates binding to the mitochondrial protein cytochrome c oxidase subunit II. Mol Cell Biol. 2004;24(16):7059-7071.

31. Allegra CJ, et al. American Society of Clinical Oncology provisional clinical opinion: testing for KRAS gene mutations in patients with metastatic colorectal carcinoma to predict response to anti-epidermal growth factor receptor monoclonal antibody therapy. J Clin Oncol. 2009;27(12):2091-2096.

32. Zhang Q, et al. SRC family kinases mediate epidermal growth factor receptor ligand cleavage, proliferation, and invasion of head and neck cancer cells. Cancer Res. 2004;64(17):6166-6173.

33. Koppikar P, et al. Combined inhibition of c-Src and epidermal growth factor receptor abrogates growth and invasion of head 
and neck squamous cell carcinoma. Clin Cancer Res. 2008;14(13):4284-4291.

34. Thomas SM, et al. Cross-talk between G protein-coupled receptor and epidermal growth factor receptor signaling pathways contributes to growth and invasion of head and neck squamous cell carcinoma. Cancer Res. 2006;66(24):11831-11839.

35. Brand TM, Iida M, Wheeler DL. Molecular mechanisms of resistance to the EGFR monoclonal antibody cetuximab. Cancer Biol Ther. 2011;11(9):777-792.

36. Wheeler SE, et al. Enhancement of head and neck squamous cell carcinoma proliferation, invasion, and metastasis by tumorassociated fibroblasts in preclinical models. Head Neck. 2014;36(3):385-392.

37. Gross ND, et al. Erlotinib, erlotinib-sulindac versus placebo: a randomized, double-blind, placebo-controlled window trial in operable head and neck cancer. Clin Cancer Res. 2014;20(12):3289-3298.

38. Sen B, Saigal B, Parikh N, Gallick G, Johnson FM. Sustained Src inhibition results in signal transducer and activator of transcription 3 (STAT3) activation and cancer cell survival via altered Janus-activated kinase-STAT3 binding. Cancer Res. 2009;69(5):1958-1965.

39. Baselga J, et al. Phase II randomized study of neoadjuvant everolimus plus letrozole compared with placebo plus letrozole in patients with estrogen receptor-positive breast cancer. J Clin Oncol. 2009;27(16):2630-2637.

40. Smith IE, et al. A phase II placebo-controlled trial of neoadjuvant anastrozole alone or with gefitinib in early breast cancer. $J$ Clin Oncol. 2007;25(25):3816-3822.

41. Dowsett M, et al. Prognostic value of Ki67 expression after short-term presurgical endocrine therapy for primary breast cancer J Natl Cancer Inst. 2007;99(2):167-170.

42. Stabile LP, Egloff AM, Zhou P, Gooding WE, Grandis JR, Bauman JE. Phase II study of dasatinib in combination with cetuximab in recurrent/metastatic head neck squamous cell carcinoma [abstract CT119]. Proc Annu Meet Am Assoc Cancer Res. 2016;76(14):supplement.

43. Edge S, Byrd DR, Compton CC, Fritz AG, Greene FL, Trotti A, eds. AJCC Cancer Staging Handbook. New York, NY: SpringerVerlag; 2009.

44. Eisenhauer EA, et al. New response evaluation criteria in solid tumours: revised RECIST guideline (version 1.1). Eur J Cancer. 2009;45(2):228-247

45. Dowsett M, et al. Assessment of Ki67 in breast cancer: recommendations from the International Ki67 in Breast Cancer working group. J Natl Cancer Inst. 2011;103(22):1656-1664.

46. Jordan RC, et al. Validation of methods for oropharyngeal cancer HPV status determination in US cooperative group trials. Am J Surg Pathol. 2012;36(7):945-954.

47. Cheung LW, et al. High frequency of PIK3R1 and PIK3R2 mutations in endometrial cancer elucidates a novel mechanism for regulation of PTEN protein stability. Cancer Discov. 2011;1(2):170-185.

48. Hochberg Y, Benjamini Y. More powerful procedures for multiple significance testing. Stat Med. 1990;9(7):811-818. 